\title{
Robotic Thyroidectomy: Facelift Approach
}

\author{
William S. Duke $\cdot$ David J. Terris
}

Published online: 11 December 2013

(C) Springer Science + Business Media New York 2013

\begin{abstract}
The past 2 decades have witnessed the most significant developments in thyroid surgery since the time of Kocher. Improved understanding of physiology, anatomy and perioperative management have resulted in faster, safer operations on an outpatient basis without the use of drains, while advances in surgical technology have permitted surgeons to explore minimally invasive techniques, resulting in less dissection, decreased postoperative discomfort, faster recovery times and smaller anterior cervical scars. Another important trend over this time has been the rise in remote access surgery, which seeks to remove the scar from the neck completely. Of these remote access techniques, the robotic facelift thyroidectomy is rapidly emerging as a safe, attractive approach to thyroid surgery for patients interested in completely avoiding a visible neck scar. A number of peer-reviewed publications have established the safety and efficacy of the approach, and as a result the technique is now performed at several centers around the world.
\end{abstract}

Keywords Thyroidectomy Robotic $\cdot$ Facelift .

Endocrine surgery $\cdot$ Minimally invasive $\cdot$ Patient selection · Patient counseling

This article is part of the Topical Collection on Minimally Invasive Endocrine Surgery.

W. S. Duke · D. J. Terris $(\bowtie)$

Department of Otolaryngology, Georgia Regents University, 1120 Fifteenth Street, BP-4109, Augusta, GA 30912-4060, USA

e-mail: dterris@gru.edu

W. S. Duke

e-mail:wduke@gru.edu

\section{Introduction}

There has been significant interest in minimally invasive neck surgery since Gagner [1] described the first endoscopic parathyroidectomy in 1996. Though his use of a completely endoscopic, $\mathrm{CO}_{2}$-assisted dissection has largely been replaced by other techniques, the possibility of limiting the cosmetic impact of the traditional Kocher thyroidectomy incision encouraged exploration into alternate approaches to accessing the thyroid compartment. This exploration diverged along two different pathways.

On one hand, minimally invasive anterior cervical approaches, such as the minimally invasive video-assisted thyroidectomy (MIVAT) popularized by Miccoli et al. [2], were developed. These procedures sought to lessen the extent of dissection and decrease the length of the cervical scar while still offering direct access to the thyroid. On the other hand were attempts to remove the scar from the visible portion of the anterior neck completely. These "remote access" techniques emerged primarily in Asian centers, where there is an increased risk of hypertrophic scarring and a premium placed on the cosmetic appearance of the neck [3, 4]. Initially reported as an endoscopic anterior chest and breast approach [5], numerous other methods were rapidly described using the axilla [6] or a combination of these sites $[7,8]$ to access the thyroid compartment.

Many of these early remote access techniques required $\mathrm{CO}_{2}$ insufflation to maintain the operative space, involved multiple incisions on the breast or chest, and were constrained by two-dimensional visualization and rigid endoscopic equipment. These factors limited their popularity outside of Asia. The application of gasless robotic technology to the axillary approach [9] finally allowed remote access thyroidectomy to become a viable option for a wider patient and surgeon population. 
However, as this novel approach spread internationally, questions began to arise about the safety of the technique and its applicability in Western practices [10-14]. Additionally, the procedure required the placement of drains and necessitated hospital admission, trends that the minimally invasive movement of the prior decade had sought to overcome. An alternate remote access approach, the robotic facelift thyroidectomy (RFT), was developed to help overcome some of the concerns and limitations associated with robotic axillary thyroidectomy [15••, 16].

\section{History of the Procedure}

As reports of complications associated with the robotic axillary thyroidectomy began to emerge (especially esophageal perforations related to the vector of approach and brachial plexopathy related to positioning), exploration began into ways to maintain the cosmetic benefit of this procedure while obviating its inherent limitations. Experiments evaluating endoscopic access routes to the thyroid compartment in a pig model had suggested that a superiorly based approach may offer the best view and fastest, most direct access to the thyroid, but at the expense of incisions being placed in cosmetically unfavorable locations [17]. Descriptions of parotidectomy [18] and excision of other superior neck masses [19] through a postauricular, modified facelift approach were associated with increased patient satisfaction when compared to more traditional incision placement. Therefore, in an effort to capitalize on the improved visualization and speed of dissection afforded by a superiorly based approach while simultaneously maintaining maximal cosmesis, investigation began into a novel facelift-approach thyroidectomy using robotic technology.

Development of the mature RFT approach began with cadaver studies assessing the feasibility of the procedure [20]. After defining the relevant anatomy and developing a reproducible algorithm to guide successful surgery, the procedure was performed on seven cadavers to evaluate the operative pocket. Morphometric analysis of the cadavers revealed that the RFT approach required $38 \%$ less dissection than the robotic axillary approach.

The first reported use of the RFT approach in patients was published in 2011, when Terris et al. [15••] presented a series of 14 patients undergoing 18 RFT procedures. One patient had a total thyroidectomy through bilateral incisions, and three patients had a second completion RFT procedure to address malignancies identified at the initial surgery. The report also outlined the selection criteria for this approach as well as a detailed description of the operative steps of the procedure. The mean total operative time was $155 \mathrm{~min}$. The first patient treated with this technique received a drain and was admitted, but all subsequent procedures were performed in the outpatient setting without need for drainage. There were two seromas and one patient with transient vocal fold weakness in this series. These conditions each resolved spontaneously without intervention. All patients reported temporary hypesthesia of the great auricular nerve that resolved within several weeks after surgery. There were no conversions to an anterior cervical approach. No patients experienced permanent vocal fold weakness, and there were no episodes of hypoparathyroidism.

A follow-up study [21••] expanded on the patient selection criteria, including suggested limitations on the maximum size of the nodule appropriate for RFT resection as well as medical contraindications (discussed below). This study also reported on an additional three patients undergoing unilateral thyroid lobectomy via RFT, none of whom experienced any complications. More than 100 procedures have now been accomplished in at least 4 centers with the same complication profile described in the original reports.

\section{Advantages/Disadvantages}

The only distinct advantage that any remote access approach to the thyroid offers over anterior cervical approaches is the absence of a visible scar. Robotic facelift thyroidectomy, however, offers several specific advantages over the axillary approaches to the thyroid. First, the relevant anatomy and route of dissection are familiar to head and neck surgeons [16]. Second, there is no risk of brachial plexus injury, as has been reported with the robotic axillary approach [10, 12]. Finally, the extent of dissection in the RFT approach is less than that of the axillary approach, permitting patients to recover as outpatients without the need for postoperative drainage [15••, 20].

Despite the many advantages of the RFT approach, the technique does have limitations. First, the vector of approach and the robotic instruments currently available do not permit bilateral thyroid surgery through a single unilateral incision. Therefore, the procedure is currently indicated only in patients for whom unilateral surgery is anticipated. A completion procedure can be performed through a contralateral second facelift incision if the initial pathology justifies total thyroidectomy.

Second, transient hypesthesia in the distribution of the great auricular nerve may be anticipated, and patients should be counseled appropriately.

Third, while it may be technically feasible, there have been no published reports of comprehensive neck dissection using the RFT approach as there has been with the axillary approach [22]. This procedure is therefore not recommended for patients who need a neck dissection [15••]. 
Finally, the procedure takes longer to complete than a lobectomy performed via an anterior cervical approach [2]. Though no direct evaluation of the impact on this increased operative time on medical costs has been performed for RFT, studies of the robotic transaxillary approach suggest that this increased time, coupled with the capital expense of the robotic system and specialized equipment, may increase the cost of the procedure significantly [23•, 24]. While reimbursement for robotic procedures is significantly higher than conventional approaches in some Asian countries [4, 14], there is no increased reimbursement in the USA.

\section{Patient Selection}

Potential candidates for RFT should meet both patient and disease criteria, designed to maximize the likelihood of surgical success and minimize the risk of complications (Table 1). These selection criteria have been previously outlined $[21 \bullet \cdot$. Patients should be generally healthy and able to tolerate general anesthesia for several hours. Patients should not be morbidly obese. However, patients who are extremely thin require very deliberate flap elevation, as there is less subcutaneous tissue to buffer any deviations from the correct plane of dissection. There should be no prior history of neck surgery. Finally, the ideal patient is highly motivated to avoid a visible cervical scar, understands the limitations and risks of the procedure, and accepts the unlikely but possible chance that conversion to an anterior approach may be required.

In addition to acceptable patient characteristics, the thyroid condition being assessed should meet certain criteria to be considered appropriate for RFT [21••]. Generally, the disease to be addressed should be one that is normally treated with unilateral surgery initially, such as an enlarging or symptomatic benign nodule or a follicular lesion of undetermined significance. The nodule size should not exceed $4 \mathrm{~cm}$ in its greatest dimension, and there should be no thyroiditis or prior history of thyroid compartment surgery. The thyroid lobe to be addressed should

Table 1 Selection criteria for robotic facelift thyroidectomy

\begin{tabular}{ll}
\hline Patient factors & Disease factors \\
\hline $\begin{array}{l}\text { Highly motivated to avoid } \\
\text { cervical scar }\end{array}$ & $\begin{array}{c}\text { Unilateral surgery recommended } \\
\text { for initial treatment }\end{array}$ \\
No morbid obesity & Largest nodule $\leq 4 \mathrm{~cm}$ \\
No prior neck surgery & No thyroiditis \\
American Society of & No pathologic lymphadenopathy \\
Anesthesiologists class 1 or 2 & No substernal extension \\
& No extrathyroidal extension
\end{tabular}

Adapted from Terris et al. $[21 \bullet \bullet]$ have no substernal component, and there should be no evidence of a high-grade malignancy such as extrathyroidal extension of the lesion or concerning lymphadenopathy.

\section{Patient Counseling}

Proper counseling and tempering of expectations is crucial to patients' perceptions of a successful surgical outcome. Of paramount importance, patients must understand that RFT carries the same risks of surgery as the anterior approaches, with the additional side effect of transient hypesthesia of the ipsilateral ear. It is also important that they understand that RFT is not minimally invasive surgery. It is associated with a longer operative time, a more extensive dissection and a longer recovery period than some anterior approaches, and it should therefore be considered more invasive than conventional surgery.

All patients should be counseled that conversion to an anterior cervical approach may be required, and they should consent to this possibility. Patients should also understand that, in its current iteration, the contralateral thyroid lobe cannot be addressed with a unilateral RFT approach. Should the final pathology reveal malignancy, a second RFT on the opposite side or an anterior cervical approach is required to remove the remaining thyroid lobe.

Finally, patients should appreciate that this is not a "scarless" operation; the scar is merely hidden behind the ear and hairline. Absence of a cervical scar is the only advantage of any remote access procedure. RFT does have the advantage over robotic axillary thyroidectomy, however, in that there is no risk of brachial plexus injury, the dissection is less extensive, and there is no need for postoperative drainage and no need for hospital admission.

\section{Procedure}

The robotic facelift thyroidectomy procedure has been

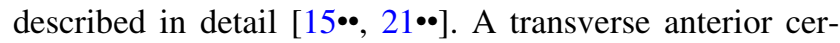
vical incision is outlined in a natural neck crease while the patient is sitting upright in the preoperative holding area. This mark is made in the unlikely event that conversion to an open procedure is necessary. The patient is then moved to the operating room and placed on the operating table. The patient should be positioned just off center of the middle of the table toward the side to be operated on. The top of the patient's head should be almost level with the top of the operating table.

General anesthesia is induced, and anesthesia is maintained with a propofol drip. This allows rapid titration of the anesthetic depth. A short-acting muscle relaxant, if necessary, is used to facilitate intubation. The patient is 
intubated with an electromyographic (EMG) endotracheal tube (ETT) to permit intraoperative laryngeal nerve monitoring. A GlideScope (Verathon Inc., Bothell, WA) is useful during intubation so that all members of the operative team can confirm appropriate positioning of the EMG electrodes. A straight extension is placed on the anesthesia circuit to limit tension on the tubing. A three-way stopcock valve connects the $\mathrm{CO}_{2}$ return tubing to the anesthesia circuit to prevent kinking of this tube. The bed is rotated $180^{\circ}$ so that the operative field is away from the anesthesia provider. The patient's arms are tucked at their sides and secured with wide silk tape. A formal safety strap that attaches to the table is not used above the patient's waist because the strap interferes with placement of the retractors. The patient's head is turned $20^{\circ}-30^{\circ}$ away from the surgical side, and the face is supported with soft towels. The anesthesia circuit is taped to the operating table to prevent any excessive tension that would cause the endotracheal tube to twist or migrate.

The occipital hairline is shaved back $1 \mathrm{~cm}$, and the facelift incision is marked. The incision begins near the inferior extent of the earlobe in the postauricular crease, and is carried superiorly and then posteriorly into the shaved region of the occipital hairline in a gentle curve that will be obscured by the auricle (Fig. 1). This placement ensures the incision will be completely concealed once the hair re-grows. The incision continues posteriorly and inferiorly as far as necessary to ensure adequate exposure.

The planned incision line is then infiltrated with $0.25 \%$ bupivacaine with 1:200,000 epinephrine, and the patient is prepped and draped in sterile fashion. The skin is incised with a knife, and a guarded electrosurgical handpiece is used to develop a subplatysmal flap until the sternocleidomastoid muscle (SCM) is identified. Dissection then continues anteriorly and inferiorly along the SCM as a

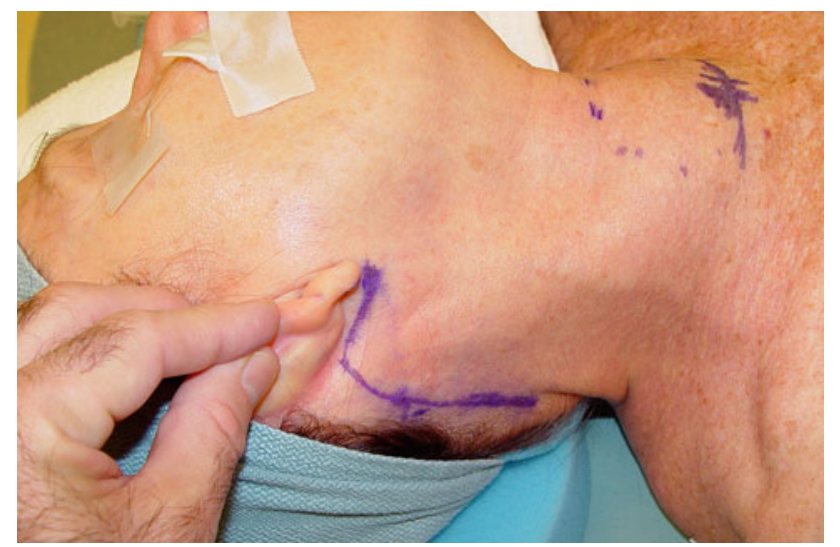

Fig. 1 The robotic facelift thyroidectomy incision begins in the postauricular crease and crosses over to the occipital hairline concealed behind the ear. The incision is placed approximately $1 \mathrm{~cm}$ within the hairline to ensure that it is invisible

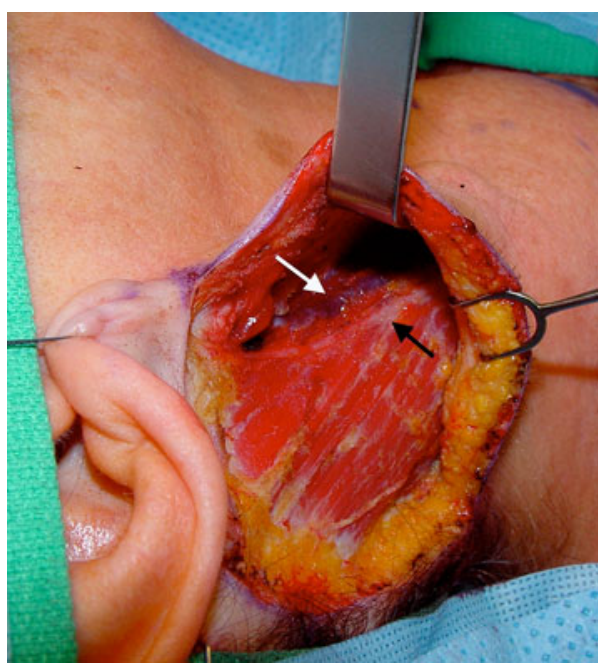

Fig. 2 The great auricular nerve (black arrow) and external jugular vein (white arrow) are identified and preserved on the surface of the sternocleidomastoid muscle

series of landmarks is encountered. The first structure identified is the great auricular nerve (GAN). Continued dissection superficial to the GAN reveals the external jugular vein and subsequently the anterior border of the SCM (Fig. 2). The external jugular vein is preserved, though it may be divided if necessary to improve exposure. Dissection continues down the anteromedial border of the SCM to the clavicle. Visualization is facilitated by placing the operating table in reverse Trendelenberg position and rotating it away from the surgeon.

A muscular triangle bordered by the SCM, the omohyoid and the sternohyoid is then defined (Fig. 3). The omohyoid, sternohyoid and sternothyroid muscles are

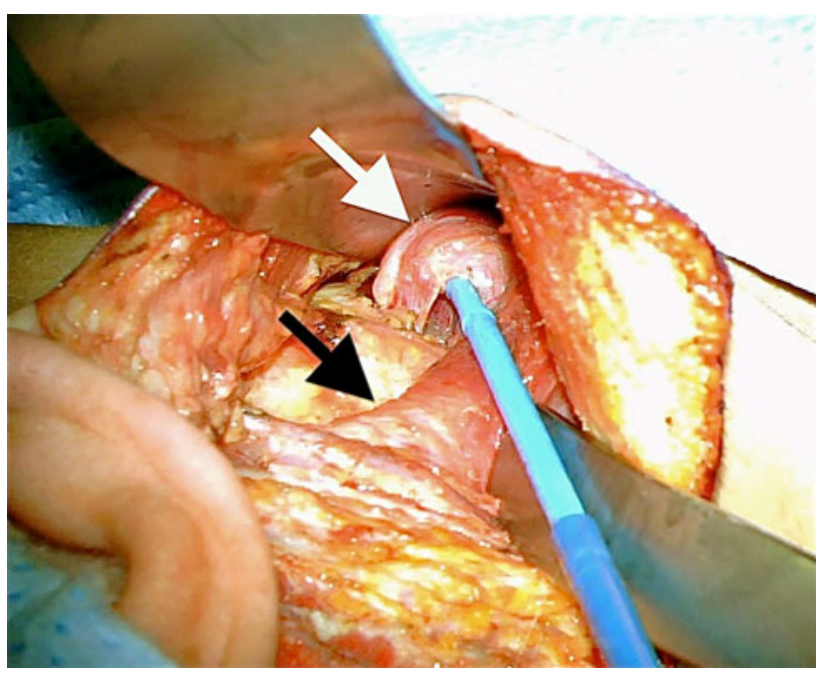

Fig. 3 The muscular triangle bordered by the anterior surface of the sternocleidomastoid muscle (black arrow), the superior border of the omohyoid muscle (white arrow) and the posterior border of the sternohyoid muscle 


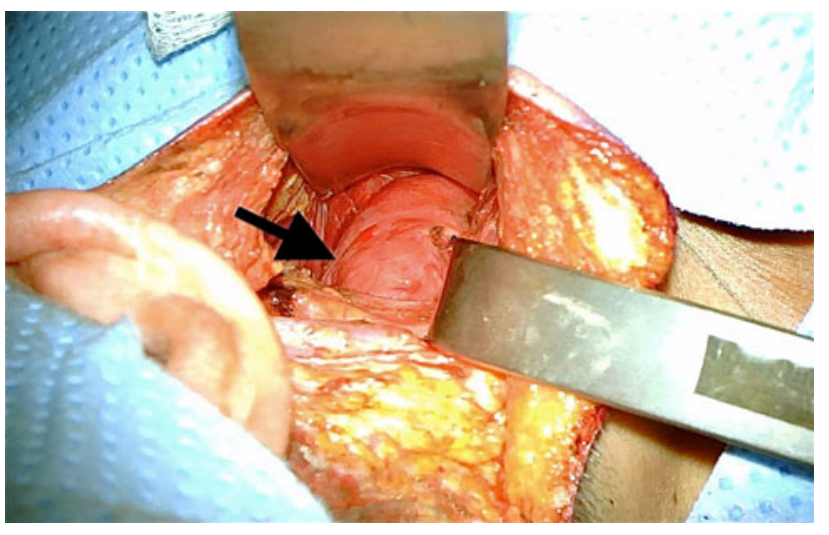

Fig. 4 The strap muscles are retracted ventrally, exposing the superior pole of the thyroid gland (arrow)

retracted ventrally to expose the superior pole of the thyroid gland (Fig. 4). The strap muscles are then elevated off of the rest of the thyroid lobe and the superior vascular pedicle is isolated. The lobe is mobilized as much as possible during this dissection. The modified Chung retractor (Marina Medical, Sunrise, FL, USA) is positioned to retract the strap muscles ventrally. The retractor system is anchored to the operating table frame on the side opposite the lobe being resected. A Singer hook (Medtronic, Jacksonville FL, USA) attached to a Greenberg retractor (Codman \& Shurtleff, Inc, Raynham, MA, USA) secured to the ipsilateral side of the table is used to retract the SCM laterally and dorsally, thereby maintaining the surgical pocket (Fig. 5). The robotic cart is then deployed.

The robotic console is positioned near the operating table opposite of the side being dissected, with the pedestal angled $30^{\circ}$ away from the table. Fine positioning adjustments are more easily accomplished by moving the table

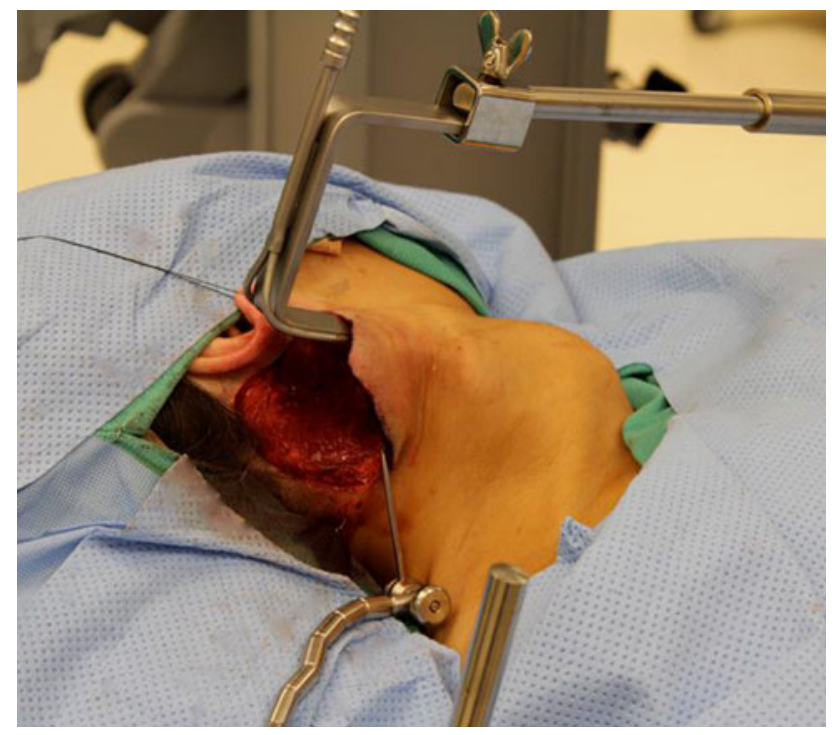

Fig. 5 The operative pocket with retractors in place rather than by moving the robot console. The camera arm with a $30^{\circ}$ down-facing endoscope is positioned first. The camera is advanced along the long axis of the modified Chung retractor. The camera arm is nearly fully extended so the elbow joints of the camera arm will not impede the movement of the other arms. A Harmonic device (Ethicon Endosurgery Inc., Cincinnati, OH, USA) is placed in the dominant arm, and a Maryland grasper is used in the nondominant arm (Fig. 6).

The robotic portion of the procedure begins by dividing the superior vascular pedicle with the Harmonic device. The superior thyroid pole is retracted inferiorly and ventrally to expose the inferior constrictor muscle. This muscle is traced inferiorly to its lower border, taking care to avoid injuring the superior laryngeal nerve. The superior parathyroid gland is identified on the posterior aspect of the thyroid and reflected away from the thyroid. The recurrent laryngeal nerve (RLN) is then identified laterally as it courses under the inferior constrictor (Fig. 7). The nerve is dissected inferiorly, exposing the ligament of Berry. With the nerve under direct visualization, the ligament is transected with the Harmonic device, and the thyroid isthmus is divided. The middle thyroid vein is then divided. The inferior parathyroid gland is bluntly dissected inferiorly away from the thyroid, and the inferior vasculature is transected with the Harmonic device. Any remaining attachments between the thyroid lobe and the surrounding soft tissue or trachea are divided, and the specimen is removed.

The robotic cart is removed. The surgical field is irrigated, and Surgicel (Ethicon, Inc., Somerville, NJ) is

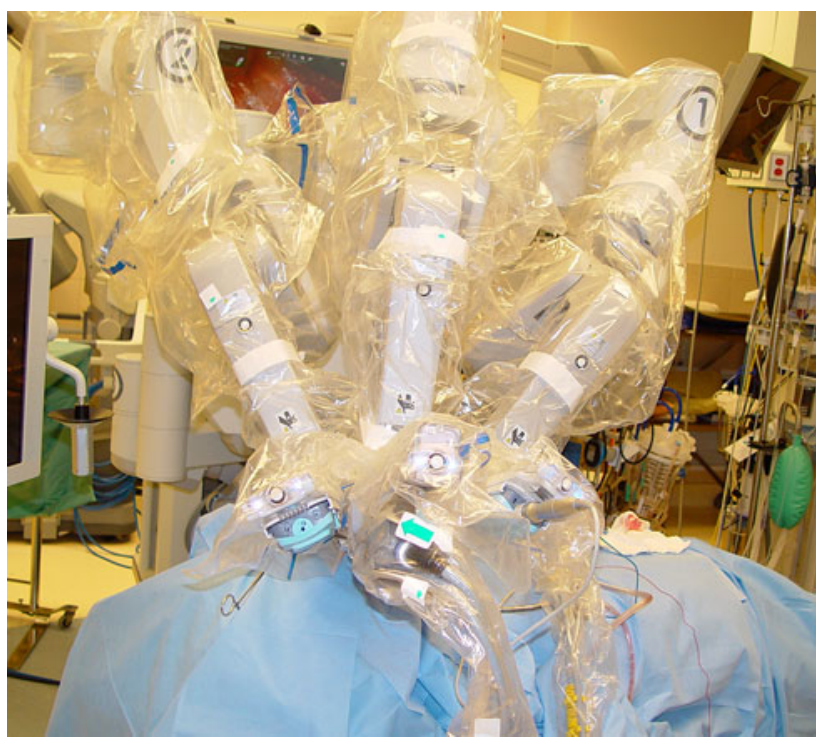

Fig. 6 The robotic console in position. A Harmonic device is placed in the dominant arm (arm 1) and a Maryland forceps is placed in the non-dominant arm (arm 2) 


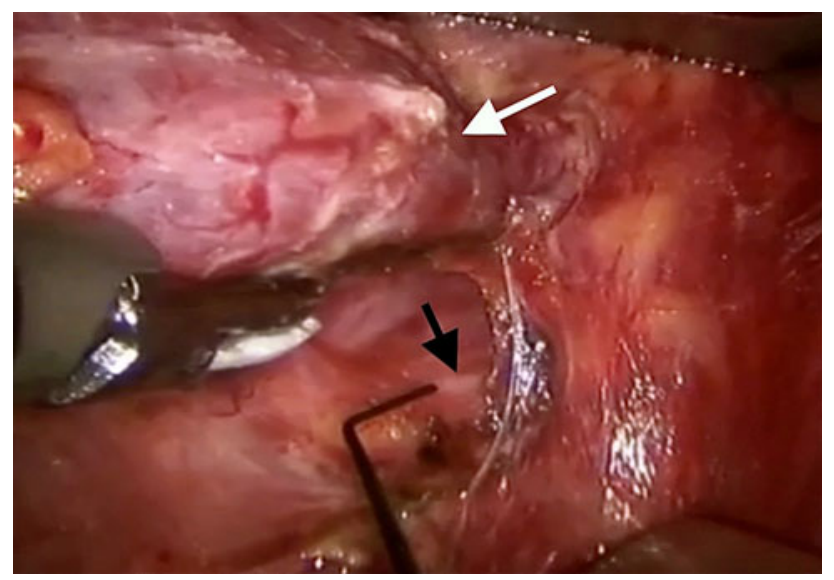

Fig. 7 The thyroid (white arrow) is retracted ventrally, and the recurrent laryngeal nerve (black arrow) is identified just inferior to the lower border of the inferior constrictor muscle, shown here being stimulated by a nerve probe

placed into the thyroid bed. The incision is approximated using buried interrupted deep dermal 4-0 Vicryl sutures (Ethicon, Inc., Somerville, NJ, USA). The skin edges are sealed with Dermaflex tissue adhesive (Chemence Medical Products, Inc., Alpharetta, GA, USA) and 1/4 inch SteriStrips (3M Corp., St. Paul, MN, USA) placed horizontally along the incision. No drains are utilized. Deep extubation is preferable to minimize coughing or straining on emergence from anesthesia.

\section{Results}

More than 60 RFT procedures have been accomplished in our center. In our most recent peer-reviewed publications, 22 RFT procedures in 18 patients were reported [15••, $21 \bullet \bullet$, 25]. The procedures were completed on an outpatient basis without drainage in all but the first patient. There was one incidence of transient vocal fold weakness and two seromas, all of which resolved without intervention. There have been no episodes of hypocalcemia and no conversions to an anterior approach. This experience has been repeated in at least four centers, with more than 100 procedures accomplished with the same complication profile as described in the original reports.

A direct comparison of RFT and robotic axillary thyroidectomy techniques has been performed [16]. The mean operative time for the first ten RFT procedures was $156.9 \mathrm{~min}$ compared to $196 \mathrm{~min}$ for the five axillary approach procedures. While these differences did not reach statistical significance in this small series, the operative time in the RFT group compared favorably with early reports of the robotic axillary approach $[9,26]$ and steadily shortened throughout the study period, while the robotic axillary times remained stable. All robotic axillary thyroidectomy patients were managed with drains as inpatients, while all but the first RFT patient were discharged on the day of surgery without drains. All patients treated with the axillary approach experienced chest wall numbness, and hypesthesia of the great auricular nerve distribution was ubiquitous in the RFT population. No major or permanent complications have been reported with the RFT approach.

\section{Conclusions}

The robotic facelift thyroidectomy has been shown to be a safe and viable option for selected individuals who place paramount importance on avoiding a cervical scar after thyroid surgery. RFT has several advantages over the robotic axillary thyroidectomy, but is currently limited to unilateral surgery. As this technique is cautiously being implemented on a broader scale, adherence to strict patient selection criteria is recommended to minimize the chance of operative failure or patient harm. A more robust picture of the full potential and limitations of this procedure will develop as more patient experiences are reported.

\section{Compliance with Ethics Guidelines}

Conflict of Interest David J. Terris has received honoraria and had travel/accommodations expenses covered/reimbursed by Johnson \& Johnson. William S. Duke declares that he has no conflict of interest.

Human and Animal Rights and Informed Consent This article does not contain any studies with human or animal subjects performed by any of the authors.

\section{References}

Papers of particular interest, published recently, have been highlighted as:

- Of importance

•- Of major importance

1. Gagner M. Endoscopic subtotal parathyroidectomy in patients with primary hyperparathyroidism. Br J Surg. 1996;83:875.

2. Miccoli P, Berti P, Conte M, et al. Minimally invasive surgery for thyroid small nodules: preliminary report. J Endocrinol Invest. 1999;22:849-51.

3. McCurdy J. Considerations in Asian cosmetic surgery. Facial Plast Surg Clin N Am. 2007;15:387-97.

4. Duh Q. Robot-assisted endoscopic thyroidectomy: has the time come to abandon neck incisions? Ann Surg. 2011;253(6):1067-8.

5. Ohgami M, Ishii S, Arisawa Y, et al. Scarless endoscopic thyroidectomy: breast approach for better cosmesis. Surg Laparosc Percutan Tech. 2000;10:1-4.

6. Ikeda Y, Takami H, Niimi M, et al. Endoscopic thyroidectomy by the axillary approach. Surg Endosc. 2001;15:1362-4. 
7. Shimazu K, Shiba E, Tamaki Y, et al. Endoscopic thyroid surgery through the axillo-bilateral-breast approach. Surg Laparosc Percutan Tech. 2003;13:196-201.

8. Choe JH, Kim SW, Chung KW, et al. Endoscopic thyroidectomy using a new bilateral axillo-breast approach. World J Surg. 2007;31:601-6.

9. Kang SW, Lee SC, Lee SH, et al. Robotic thyroid surgery using a gasless, transaxillary approach and the da Vinci S system: the operative outcomes of 338 consecutive patients. Surgery. 2009;146:1048-55.

10. Kuppersmith R, Holsinger F. Robotic thyroid surgery: an initial experience with North American patients. Laryngoscope. 2011;121:521-6.

11. Perrier N. Why I have abandoned robot-assisted transaxillary thyroid surgery. Surgery. 2012;152:1025-6.

12. Landry C, Grubbs E, Warneke C, et al. Robot-assisted transaxillary thyroid surgery in the United States: is it comparable to open thyroid lobectomy? Ann Surg Oncol. 2012;19:1269-74.

13. Kandil E, Noureldine S, Yao L, Slakey D. Robotic transaxillary thyroidectomy: an examination of the first one hundred cases. J Am Coll Surg. 2012;214:558-66.

14. Dionigi G. Robotic thyroidectomy: Seoul is not Varese. Otolaryngol Head Neck Surg. 2013;148:178

15. • Terris DJ, Singer MC, Seybt MW.: Robot facelift thyroidectomy: II. Clinical feasibility and safety. Laryngoscope 2011, 121:1636-1641. This paper describes the operative steps of the RFT procedure and the results of the first clinical experience with RFT.

16. Terris DJ, Singer MC. Qualitative and quantitative differences between 2 robotic thyroidectomy techniques. Otolaryngol Head Neck Surg. 2012;147(1):20-5.

17. Terris DJ, Haus BM, Nettar K, et al. Prospective evaluation of endoscopic approaches to the thyroid compartment. Laryngoscope. 2004;114:1377-82.
18. Terris DJ, Tuffo KM, Fee WE Jr. Modified facelift incision for parotidectomy. J Laryngol Otol. 1994;108:574-8.

19. Roh JL. Retroauricular hairline incision for removal of upper neck masses. Laryngoscope. 2005;115:2161-6.

20. Singer MC, Seybt MW, Terris DJ. Robot facelift thyroidectomy: I preclinical simulation and morphometric assessment. Laryngoscope. 2011;121:1631-5.

21. • Terris D, Singer MC, Seybt MW.: Robotic facelift thyroidectomy: patient selection and technical considerations. Surg Laparosc Percutan Tech 2011, 21(4):237-242. This paper provides selection criteria for RFT patients and a detailed description of the operative steps.

22. Kang SW, Lee SH, Ryu HR, et al. Initial experience with robotassisted modified radical neck dissection for the management of thyroid carcinoma with lateral neck node metastasis. Surgery. 2010;148:1214-21.

23. - Cabot JC, Lee CR, Brunaud L, et al.: Robotic and endoscopic transaxillary thyroidectomies may be cost prohibitive when compared to standard cervical thyroidectomy: a cost analysis. Surgery 2012, 152:1016-1024. This paper provides a cost assessment of robotic thyroidectomy.

24. Inabnet WB. Robotic thyroidectomy: must we drive a luxury sedan to arrive at our destination safely? Thyroid. 2012;22:988-90.

25. Terris DJ, Singer MC. Robotic facelift thyroidectomy: facilitating remote access surgery. Head Neck. 2012;34:746-7.

26. Stevenson CE, Gardner DF, Grover AC. Patient factors affecting operative times for single-incision trans-axillary robotic-assisted (STAR) thyroid lobectomy: does size matter? Ann Surg Oncol. 2012;19:1460-5. 